\title{
Interacting Light Paths Attract KELEA (Kinetic Energy Limiting Electrostatic Attraction) and Can Lead to the Activation of Water
}

\author{
W. John Martin \\ Institute of Progressive Medicine, South Pasadena, CA, USA \\ Email: wjohnmartin@ccid.org
}

Received 24 September 2015; accepted 25 October 2015; published 28 October 2015

Copyright (C) 2015 by author and Scientific Research Publishing Inc.

This work is licensed under the Creative Commons Attribution International License (CC BY). http://creativecommons.org/licenses/by/4.0/

(c) (i) Open Access

\begin{abstract}
Water can acquire a kinetic activity, which is attributed to the absorption of an environmental force termed KELEA (kinetic energy limiting electrostatic attraction). This activity can lead to increased volatility of the water that can be measured as the progressive weight loss in closed but not completely sealed containers. It has been proposed that KELEA is a natural force required to prevent the fusion and possible annihilation of electrostatically attracted opposite electrical charges. As such, it may be especially available where there is a convergence of force fields of opposing electrical charges. At least conceptually, this may arise with facing light sources with centrally directed light beams. An oscillating attraction and release of KELEA may be facilitated by repetitively disrupting the light paths using an overhead flashing light source. This paper reports on preliminary studies that are based on this premise. Although not proving the premise, the reported experiments do indicate a simple method for activating water. Moreover, the described procedure should allow for further exploration of the underlying mechanism of water activation. The procedure involves the use of four, diagonally placed regular LED traffic lights, with an overhead strobe light. The volatility of water samples in closed but not completely sealed glass vials placed within the lighted area significantly increases in a manner that persists well beyond the periods of light exposure. The paper further shows that activated water can indirectly lead to the activation of nearby water. The reported observations are of both practical and theoretical importance.
\end{abstract}

Keywords

ACE, Alternative Cellular Energy, KELEA, Water, Hydrogen Bonding, Light 


\section{Introduction}

Progress in complementary alternative medicine has been hampered by the lack of a unifying hypothesis that can explain the many observed health benefits. Cells require energy to perform their various functions and many illnesses can be attributed to an insufficiency of cellular energy (ICE). It is generally assumed that apart from photosynthesis in plants and in certain bacteria, cellular energy is obtained solely through food metabolism [1]. This view has been challenged with the description of a third or alternative cellular energy (ACE) pathway [2]. It has been proposed that this pathway is expressed as a dynamic (kinetic) quality of the body's fluids that results from the absorption of an external force termed KELEA (kinetic energy limiting electrostatic attraction) [2]. A fundamental role of this force may be to prevent the fusion and possible annihilation of opposite electrical charges when they are being increasing electrostatically attracted to one another [2]. KELEA may also explain the repulsion of like electrical charges. Experimental studies indicate that KELEA can also reduce the strength of intermolecular hydrogen bonding in water [3], enabling the water molecules to better support metabolic and other cellular functions [4].

KELEA activation of water and other fluids results in a range of changed physical properties. Among these are lower surface tension, reduced specific heat and increased volatility of the fluids [5]. Repeated measurements of the weight of closed but not completely sealed water containing vials provide a reliable method of comparing the volatility of different water samples [3]. This method is used in the present study.

Electromagnetic radiation comprises the forward linear movements of photons that create coordinated, fluctuating electrical and magnetic changes [6]. The electrical and magnetic fields are synchronized in intensity, although directionally at right angles to one another. The electrical fields of converging light paths are likely to impose interactive electrical fields that can attract KELEA. Periodic disruption of the light paths can lead to the release of KELEA and its potential transfer to nearby water. In other words, increasing the proximity of photon induced opposite electrical charges may lead to the activation of nearby fluids.

The consideration of this possible scenario is prompted by historical reports of clinical benefits from various fluctuating electrical devices [7]. Oscillating electrical devices may provide for the direct capturing and release of intensified KELEA. They can also potentially establish resonant coupling with electrically inducible nearby objects, resulting in forced interaction between electrical charges coming from opposite directions [8]. The author is also familiar with a system that comprises multiple computers with facing screens (www.eesystem.com). The screens are supposedly transmitting coded healing messages, but proof on this presumption has not been provided. It is possible that any opposing and/or converging sources of light, especially if the light rays are subjected to variable disruptions, will provide a useful and simple means for KELEA activation of nearby water.

After a series of preliminary studies, a system was constructed by using a set of 4 diagonally placed green LED traffic lights with a flashing overhead strobe light. This system was tested for its capacity to achieve a prolonged increase in the volatility of water; being a presumptive measure of continued KELEA absorption by the water.

\section{Materials and Method}

Water in Glass Vials: Before beginning the experiment, all residual vials of activated water from previous experiments were removed from the room containing the weighing balance and from a non-adjacent room in which the lights were to be placed. Thirty-one previously unused $1.25 \mathrm{oz}$ glass vials with screw caps were purchased from the Container Store, Pasadena, CA. The vials were brought into the weighing room, numbered and individually weighed using a Sartorius Balance that reads to $0.1 \mathrm{mg}$. A gallon of distilled water (Arrowhead) was purchased from a local store on the day of the experiment. Using an open handle measuring cup, each vial was near completely filled with water and capped tightly by hand. The water filled vials were let stand for 5 hours before being individually weighed. The numbered vials were reweighed 12 hours later. Ten test vials were selected at random without regard to any of the earlier measurements. Five of the vials were for the study described in this paper (test vials) and five vials were used for another study that will be reported elsewhere. The remaining 21 water containing vials provided the control vials for both experiments.

Lights: Four 12" diameter green LED traffic lights, catalogue number DR6-GCFB-VLA, were purchased directly from General Electric Inc. (Amesbury, MA). They were arranged in an inwardly facing square using the cardboard packaging in which the lights were supplied. The exposed convex covering of the lights protruded into the square space, such that the minimal separation between facing lights was 7”. The wires from the lights were connected to separate electrical plugs, which were inserted into a multi-outlet electrical extension cord. A 5" 
diameter, $25 \mathrm{~W}$ strobe light (Stroboscope Plus, AMScan.com, Elmsford NY) was placed above the traffic lights so that its bulb was 18 " higher than the central point of the illumination by the traffic lights. For the present experiment, the strobe light was set at its maximum flash rate of 10 times per second. The five test vials of water were randomly placed upright on the ground between the four traffic lights and below the flashing strobe light.

Light Exposure and Weighing Times: The beginning time of exposure of the vials to the traffic lights was taken as time zero. The test vials were temporarily removed from the room with the traffic lights for periodic weighing. This was done at hourly intervals for the first 6 hours from time zero and, thereafter, at 2 hour intervals for an additional 6 hours. After weighing, the vials were randomly replaced within the lighted area. The control vials were also weighed, using the same time schedule. Following the initial 12 hours of light exposure, minus the approximately 5 minute interruptions required for weighing, the test vials remained in the weighing room for 12 hours. The test vials were then re-exposed to the traffic lights for a second 12-hour period, with periodic removal for weighing. The test vials were, thereafter, left in the weighing room within 2 feet of the control vials. After the 48-hour mark, 4 of the control vials were removed from this particular study to help answer auxiliary questions. The test vials and the remaining control vials were weighed at 12 hour intervals over the next 12 days. The final weight measurements were obtained on the 5 test vials and the remaining control vials on days 34 and 35 days (840 hours) after time zero.

Presentation of Data: The cumulative change in weight from time zero was measured in $\mathrm{mg}$ for all of the available control and test vials throughout a 35-day experiment. Particular emphasis was placed on the initial 48 hours, which covered the two 12-hour periods of exposure to the traffic lights ( 0 - 12 hours and 24 - 36 hours) and two periods with no traffic light exposure (12 - 24 hours and 36 - 48 hours). For ease of presentation and because of the relatively little variability among the control vials, the average value, along with the highest and lowest control reading at each time points are shown in Table 1. For the 5 test vials the average and actual values are shown for each time point.

\section{Results}

The empty vials with caps weighed an average of 28.02 grams (gm) prior to being filed with water (range 27.88 to 28.24). With added water, the vials weighed an average of $63.590 \mathrm{gm}$, with the measured amounts of added water ranging from 34.56 - $37.09 \mathrm{ml}$. Upon re-weighing the individual vials 12 hours later, the average weight of the tightly capped vials was essentially unchanged (63.590 gm). Matched comparisons of the weights of each of the 31 individual vials at the two time points showed slight variability of from minus $1.1 \mathrm{mg}$ to plus $0.7 \mathrm{mg}$. This degree of occasional minor variation was attributed to not quite waiting long enough for the balance readings to fully stabilize. The finding of no appreciable weight change in the tightly closed vials containing untreated water was anticipated since it was consistent with prior observations in other experiments. The decision was made to proceed with the traffic lights experiment using 5 randomly selected test vials. Another 5 vials were assigned to a different experiment, the results of which will be presented elsewhere. Twenty-one vials were left as the control vials for both experiments.

Table 1 includes the data acquired from the 21 control vials and from the 5 test vials during the first 12-hour period, that is, during the time in which the test vials were initially exposed to the traffic lights. Even after 1 hour of exposure to the traffic lights, an appreciable loss of weight had occurred in each of the 5 test vials, but not in the control vials. The weight loss in the test vials progressively increased over the later time points. There was actually a slight increase in the average recording of the weights of the control vials, measured as minus 0.5 $\mathrm{mg}$ (The slight increase was within the range of variability occasionally encountered in repeated weighing). The same small but discernable decrease in weight compared with the time zero recorded weights was seen with most of the control vials in the measurements made up to hour 8 . At hour 10 and, thereafter, a progressive reduction in the average weight of the control vials, compared to the weight at time zero was observed. The level of weight loss was far less than that in the test vials. Thus, the average cumulative loss of the test vials at 12 hours was $32.2 \mathrm{mg}$, whereas it was only $1.5 \mathrm{mg}$ in the control vials.

The test vials were not exposed to the traffic lights during the second 12-hour period. Still the cumulative weight losses of the test vials in the second 24-hour period, although proceeding at a slower rate than during the first 12-hour period, still exceeded those of the control vials in the second 24-hour period (41.5 mg compared to $2.8 \mathrm{mg}$ ). The average weight loss of the control vials that was first clearly identified at hour number 10, continued during the second 12-hour period reaching $2.8 \mathrm{mg}$ at 24 hours. 
Table 1. Activation of water samples using exposure to interacting light paths.

\begin{tabular}{ccccccccccccccccc}
\hline \multirow{2}{*}{$\begin{array}{c}\text { Control vs. } \\
\text { Test Vials }\end{array}$} & \multicolumn{1}{c}{ Cumulative Change in Weight (mg) of Vials at the Time in Hours after Beginning the Initial Light Exposure } \\
\hline Control Av $^{*}$ & $-\mathbf{0 . 5}$ & 2 & 3 & 4 & 5 & 6 & 8 & 10 & 12 & 24 & 36 & 48 & $84^{\wedge}$ & 816 & 840 \\
Lowest & -0.2 & -1.1 & -1.2 & -1.3 & -1.4 & -1.5 & -1.5 & -0.1 & -0.1 & 0.0 & -0.3 & 1.3 & 1.3 & 77.1 & 79.6 \\
Highest & -0.9 & 1.2 & 1.5 & 2.1 & 2.1 & 3.1 & 3.6 & 6.1 & 6.4 & 8.9 & 10.4 & 12.9 & 15.8 & 181.1 & 185.4 \\
Test Average & $\mathbf{1 . 9}$ & $\mathbf{3 . 8}$ & $\mathbf{6 . 0}$ & $\mathbf{9 . 3}$ & $\mathbf{1 2 . 2}$ & $\mathbf{1 5 . 4}$ & $\mathbf{2 1 . 8}$ & $\mathbf{2 8 . 0}$ & $\mathbf{3 2 . 2}$ & $\mathbf{4 1 . 5}$ & $\mathbf{6 6 . 9}$ & $\mathbf{7 6 . 3}$ & $\mathbf{8 7 . 4}$ & $\mathbf{3 6 2 . 0}$ & $\mathbf{3 6 9 . 1}$ \\
Test Vial 1 & 2.1 & 3.6 & 5.9 & 9.2 & 11.8 & 14.9 & 21.1 & 26.5 & 32.2 & 46.2 & 71.4 & 81.4 & 90.9 & 334.5 & 340.9 \\
Test Vial 2 & 1.2 & 3.6 & 5.8 & 8.9 & 11.5 & 14.4 & 20.6 & 26.9 & 30.1 & 38.2 & 56.3 & 64.0 & 76.8 & 381.9 & 390.3 \\
Test Vial 3 & 2.2 & 3.4 & 5.5 & 8.2 & 11.4 & 14.6 & 21.5 & 28.7 & 34.0 & 43.8 & 73.8 & 84.7 & 91.7 & 298.5 & 303.7 \\
Test Vial 4 & 2.0. & 4.0 & 6.3 & 10.0 & 13.2 & 16.8 & 23.9 & 30.1 & 34.7 & 45.1 & 77.1 & 89.5 & 103.8 & 376.5 & 382.4 \\
Test Vial 5 & 1.7 & 4.4 & 6.7 & 10.3 & 13.1 & 16.2 & 22.0 & 27.6 & 29.8 & 34.3 & 55.8 & 62.0 & 73.6 & 418.7 & 428.4 \\
\hline
\end{tabular}

"Average weight of the control vials; "The negative values indicate that the measured weights of some of the control vials exceeded the weights recorded at time zero; 'Seventeen control vials were available at this and at the later time, since 4 had been used in a side study.

The traffic light exposure was resumed for the test vials during the third 12-hour period (24 - 36 hours). This period resulted in an increased weight loss by the test vials (66.9 $\mathrm{mg}$ at hour 36 compared to $41.5 \mathrm{mg}$ at hour 24). This was comparable to the weight loss that occurred in the test vials during the first 12-hour period (32.2 mg) and was greater than the weigh loss of the test vials during the second 12-hour period (41.5 mg minus $32.2 \mathrm{mg}$, i.e. $9.3 \mathrm{mg}$ ). The control vials also showed continuing weight loss over the third 12-hour period (3.2 $\mathrm{mg}$ minus $2.8 \mathrm{mg}$ ) but less than that during the second 12-hour period (2.8 mg minus $1.5 \mathrm{mg})$.

There was no traffic light exposure of the test vials during the fourth 12-hour period. Yet there was still far more weight loss in the test vials compared to the control vials $76.3 \mathrm{mg}$ minus $66.9 \mathrm{mg}$; compared to $4.8 \mathrm{mg}$ minus $3.2 \mathrm{mg}$.

Weight loss of the test and the control vials continued over the course of the experiment. The time points shown in Table 1 are representative of many of the other time points at which the vials were weighed. The data indicate the continuing activation of the water in both the test and the control vials. The 816 and 840 hours time points are shown in Table 1 to confirm the continuing weight loss of both the test and the control vials over the final 24 hours of the experiment. The data also indicate that the rate of weight loss of the control vials had increased somewhat over time, while that of the test vials had decreased, although it was still greater than that of the control vials.

\section{Discussion}

The three basic findings of this paper are: 1) That capped vials of distilled water placed in the vicinity of converging and opposing light paths provided by a set of 4 green LED traffic lights, with an overhead flickering strobe light, will lose weight when compared to control vials of the same water not exposed to the light paths; 2) That the weight loss of the traffic light exposed test vials, which is viewed as a measure of kinetic activation of the water, continues well beyond the two periods of time that the vials were exposed to the lights; 3) That control vials also begin to lose weight several hours into the experiment.

The interpretation of this third finding is that the weight loss in the control vials reflects a low level of indirect water activation from maintaining vials of activated water in the same room as the control vials of water. It is clear that there was no significant weight loss in any of the vials for the 12 hours that preceded the beginning of the test procedure. Although the results are not included in this paper, a parallel energy exposure study was performed on the 5 other vials of water during the first several hours of the light study. The water in these vials clearly became activated as evidenced by reduction in weight. These vials of activated water were then left in the weighing room, as were the traffic light exposed vials; except during the two 12-hours periods of exposure to 
the traffic lights. It is noteworthy that the average weight loss in the control vials was less during the second 12-hour period of light exposure of the test vials, than during the preceding or the following 12 hour periods. Conversely, the weight loss of the test vials exposed to the traffic lights was greater during the second 12-hour period of exposure to the traffic lights than during the preceding or the following 12 hour periods. This is consistent with the notion that once water is activated, it may attract KELEA and transfer some of the energy to nearby water [4].

An understanding that activated water is able to indirectly activate other water without direct contact formed the basis of Johann Grander's water activating device (www.grander.com). This approach is also being employed in a Los Angeles bottled water plant that has a floating sealed container of activated water in the major water tank, from which the bottles are filled. This current practice, although only achieving a relatively low level of water activation, has a regulatory advantage over those activation methods, which require adding water activating compounds to the water. This is even though, once the compounds achieve a readily discernable activation of the water, they can be removed by filtration, distillation or repeated dilutions [4].

The primary goal of this paper was to determine if converging and opposing light paths could lead to a practically useful and informative method of water activation. Preliminary studies had supported the substantial improvement $(\sim 50 \%)$ in water activation by using the strobe light above the traffic lights. Many other variable features, including the use of reflected sunlight and battery driven flashlights, rather than the traffic lights are beginning to be explored. In principle, the method may work with all regions of the electromagnetic spectrum, not just visible light.

Using the present system, the weight loss consequent to light exposure far exceeded that of the control vials and was apparent even after the first hour of the initial 12-hour period of exposure to the traffic lights. An activating effect of the light, reflected in increased weight loss, was confirmed by the accelerated weight loss during the second period of light exposure. The finding that once it is begun, the water activating process can continue for an extended period of time, is consistent with the proposed self-activating capacity of initially activated water [4]. Possibly due to loss via vaporization of some of the more kinetically activated water molecules, the overall level of activation tends to remain rather stable, with a tendency to become less over time. Conversely, in completely sealed containers, the level of fluid activation has in other experiments been shown to increase over time [3].

Another goal of the paper was to demonstrate the utility of the weight loss assay to pursue various issues relating to KELEA. The decision to use 21 control samples to match against the 5 test samples was intended to underscore the reliability and robust nature of the assay system. Differential minor variations in weight measurements of various items over time have been observed and suggest the intriguing possibility of subtle influences on measured weight that have yet to be explained. A further observation in performing long-term repeated measurements of the weight of activated fluids is the variability that can occur in the rate of weight loss. This may reflect variations in the natural levels of KELEA over time [3]. The possible relationship between KELEA and other previously identified subtle forces, such as scalar energy described by Konstantin Meyl [8] and others; radiant energy described by Nikola Tesla [9]; ODIC energy described by Karl von Reichenbach [10]; and orgone energy described by Wilhelm Reich [11], is being considered.

The results presented in this paper have practical value as an added means of activating water. As described elsewhere, KELEA activated water can provide substantial agriculture [12] and industrial benefits [5], when compared to regular water. So called homeopathic remedies with proven therapeutic benefits, such as Enercel®, are essentially activated water [4]. Broadly based clinical benefits attributed to many other substances, including moringa oleifera [13] and humic/fulvic acids, may result from the apparent capacity of these compounds to activate water, either for consumption or by direct actions on the body's water. Similarly, converging and opposing light paths could potentially be directly applied to humans or animals. Indeed, claims have been made for a therapeutic benefit of a system of multiple computers installed in a therapy room (www.eesystem.com). Up to 16 pairs of facing computers are said to display proprietary coded messages of healing intentions. Simple traffic lights with repeatedly interrupted light paths may provide a more rational and less expensive alternative to the computer generated proprietary images.

Fluid activation has also been extended to other fluids, including gasoline [3], with apparent benefit in terms of fuel consumption and less toxic exhaust. Activation of water is also being investigated as a means of removing contaminants from toxic waste waters (unpublished).

An interesting system, called Aquapol, that presumably attracts and releases KELEA, has been developed by 
Wilhelm Mohorn (http://www.aquapol.co.uk). It comprises a base plate supporting outwardly projecting antennas. When placed in the ceiling of buildings, it can slowly lead to the reversal of upward water flow into the foundations and lower walls of old buildings. Again, although a small amount of electricity is required for the lights, the system described in this paper may provide a more effective means of reversing damage from the upward seepage of water into buildings. This topic also relates to the question that if KELEA activated water has biological benefits, why would Nature not render all water as being activated? A possible answer is that tightly hydrogen-bonded water is required for water to be drawn to the tops of large trees [14]. This presumably could not occur if all of the available water to tall trees were to be activated. This issue is being explored in agricultural studies documenting the benefits of KELEA activated water on food crops [12].

This reasoning is also consistent with a postulated variable role of the fluctuating electrical activity of the brain as an antenna for attracting KELEA from the environment and transferring the energy to the body's water [15]. The working hypothesis is that for some individuals, consuming KELEA activated water or being exposed to KELEA generating devices may indeed act in a positive manner to enhance the KELEA absorbing activity of the brain. The system described in this paper may help facilitate such studies.

\section{Conclusion}

A system of interacting lights is described that is able to increase the volatility of water in tightly closed but not completely sealed container vials. The modification is attributed to a loosening of the intermolecular hydrogen bonding between water molecules due to the absorption of an environmental force termed KELEA (Kinetic Energy Limiting Electrostatic Attraction). The converging and opposite light paths are viewed as situations in which opposite electrical charges move into closer proximity to each other than in a single light path. This proximity is thought to attract KELEA as part of a basic mechanism to ensure the lack of fusion and possible annihilation of the electrostatically attracted, opposite electrical charges. Repetitive disrupting of the light paths, using a strobe light in the present set of experiments, can seemingly lead to the release of the attracted KELEA and its transfer to nearby water. This paper provides data consistent with this interpretation.

\section{Acknowledgements}

The Institute of Progressive Medicine is a component of MI Hope Inc., a non-profit public charity.

\section{Conflict of Interest}

None.

\section{References}

[1] Wells, J.C. (2013) Obesity as Malnutrition: The Dimensions beyond Energy Balance. European Journal of Clinical Nutrition, 67, 507-512. http://dx.doi.org/10.1038/ejcn.2013.31

[2] Martin, W.J. (2014) Stealth Adapted Viruses; Alternative Cellular Energy (ACE) \& KELEA Activated Water. Author House, Bloomington, 321.

[3] Martin, W.J. (2015) KELEA: A Natural Energy That Seemingly Reduces Intermolecular Hydrogen Bonding in Water and Other Liquids. Open Journal of Biophysics, 5, 69-79. http://dx.doi.org/10.4236/ojbiphy.2015.53006

[4] Martin, W.J. (2015) Therapeutic Potential of KELEA Activated Water. International Journal of Complementary \& Alternative Medicine, 1, Article ID: 00001.

[5] Martin, W.J. (2015) Improved Efficiency of Heat Exchange Using KELEA Activated Water. Open Journal of Energy Efficiency, 4, 36-43.

[6] Garg, A.K. (2012) Classical Electromagnetism in a Nutshell. Princeton University Press, Princeton, 712.

[7] Titus, E.C. (1910) Standardization of High Potential Electric Currents. Transactions of the American Electro-Therapeutic Association, 19, 17-48.

[8] Meyl, K. (2012) DNA and Cell Resonance: Magnetic Waves Enable Cell Communication. DNA and Cell Biology, 31, 422-426. http://dx.doi.org/10.1089/dna.2011.1415

[9] Tesla, N. (1901) Method of Utilizing Radiant Energy. United States Patent 685958.

[10] Reichenbach, K. (1854) The Mysterious Odic Force. Translated by Korth, L.O. (1977), Aquarian Press, Wellingbo- 
rough, United Kingdom, 93.

[11] Reich, W. (1948) The Cancer Biopathy. The Discovery of the Orgone, Translated by White, A., Higgins, M. and Raphael, C.M. (1973), Vol. 2, Farrar, Straus and Giroux, New York, 433. http://www.wilhelmreichtrust.org/cancer_biopathy.html

[12] Martin, W.J. (2014) KELEA Activated Water Leading to Improved Quantity \& Quality of Agricultural Crops. Advances in Plants \& Agriculture Research, 2, Article ID: 00033.

[13] Martin, W.J. (2015) Do the Benefits of Moringa oleifera Trees Extend to KELEA Activation of Water? Advances in Plants \& Agriculture Research, 2, Article ID: 00036.

[14] Wei, C., Steudle, E. and Tyree, M.T. (1999) Water Ascent in Plants: Do Ongoing Controversies Have a Sound Basis? Trends in Plant Science, 4, 372-375. http://dx.doi.org/10.1016/S1360-1385(99)01466-1

[15] Martin, W.J. (2015) Is the Brain an Activator of the Alternative Cellular Energy (ACE) Pathway? International Journal of Complementary \& Alternative Medicine, 1, Article ID: 00002. 